\title{
Why the Vagueness Paradox is Amazing
}

\author{
Bryan Frances
}

Forthcoming in Think

\begin{abstract}
:
One of the hardest problems in philosophy, one that has been around for over two thousand years without generating any significant consensus on its solution, involves the concept of vagueness: a word or concept that doesn't have a perfectly precise meaning. There is an argument that seems to show that the word or concept simply must have a perfectly precise meaning, as violently counterintuitive as that is. Unfortunately, the argument is usually so compressed that it is difficult to see why exactly the problem is so hard to solve. In this essay I attempt to explain just why it is that the problem-the sorites paradox-is so intractable.
\end{abstract}

According to the view I christen sharpism, when Joe says to his daughter in a perfectly ordinary context 'The earth is super-duper old', his claim has an incredibly discriminating meaning: although it has one truth status if the earth is over 3,347,342,343 years, 2 days, and 17 nanoseconds old, if the earth is even a nanosecond younger then his claim has some different truth status-but we leave open what that new status might be: false, indeterminate, indeterminately indeterminate, meaningless, just under $100 \%$ true, or whatnot. The material point is that the claim changes in truth status with a nanosecond change in the earth's age. The sharp cutoff might not be a cutoff separating the true from the false, but it is a sharp cutoff nonetheless. It has this sensitive meaning even though Joe has never made any fancy linguistic definitions and doesn't even know what a nanosecond is. Another example: when I say to a visiting friend, 'The restaurant is a short walk from here', my claim has one alethic status only if the restaurant is no more than 123 meters, 6 centimeters, and 16 nanometers away. Hence, if it turns out that we were 123 meters, 6 centimeters, and 17 nanometers away from the restaurant, my claim had some different truth status. 
You might be thinking to yourself 'Why would anyone accept a view as crazy as sharpism?' The incredible truth is that there is an extremely strong argument for sharpism, one that can be illustrated with a story (cf. Horgan 1994, Williamson 1994, Priest 2003).

A few days before Halloween you are walking on Farmer Fred's farm, on your way to his pumpkin patch. Your niece wants to pick out a pumpkin to take home and carve. You say to her, in an obviously apt and relevant circumstance, 'There is a pumpkin by the tree'. You weren't able to see any pumpkins there, as the view was blocked by a shed, but you remember from last year that there were pumpkins near that huge tree you see towering behind the shed.

Suppose you uttered that sentence to your niece at noon. Given that there are such things as pumpkins, you spoke truly: just as you said, there was a living pumpkin by the very tree you indicated at noon. Call the situation you were in at that time $S_{1}$; so 'There is a pumpkin by the tree' is true when evaluated with respect to $S_{1} . S_{1}$ is the whole situation you were in at noon on that day: it's a snapshot of what the world was like at a certain instant in time.

Now I want you to imagine if things had been ever so slightly different: imagine that the top of the pumpkin had had one fewer electron in it. Call that alternative imaginary situation, with the single microscopic difference from $S_{1}, S_{2}$. Our question is this: was there a pumpkin by the tree in $S_{2}$ ?

Obviously, it's very reasonable to think that the answer is 'yes, of course'. The only difference between $S_{1}$ and $S_{2}$ is the absence of a single microscopic particle. So, given that there was a pumpkin in $S_{1}$, there was one in $S_{2}$.

Now imagine situation $S_{3}$, which is exactly like $S_{1}$ except that the top of the pumpkin has two fewer microscopic particles in it. Our new question is this: was there a pumpkin by the tree in $\mathrm{S}_{3}$ ? And of course, just as before with $S_{2}$, it's very reasonable to think that the answer is 'yes': there was a pumpkin by the tree in $S_{3}$, as the pumpkin is different by a mere two electrons off its top.

Repeat the process over and over: each time consider a pumpkin with one fewer particle in it, starting from the top and working down. For each imaginary S situation consider the question 'Is there a pumpkin by the tree in the new situation?' Another way of doing the very same thing: for each S 
consider the pumpkin claim 'There is a pumpkin by the tree' and ask yourself whether it's true. We are evaluating the pumpkin claim with respect to many zillions of situations, the Ss.

One could do this literally a trillion times and the first and the trillionth situations, $\mathrm{S}_{1}$ and $\mathrm{S}_{\text {trillion, would }}$ still look identical to the naked eye: a difference of a trillion particles from the top of a pumpkin would be literally undetectable without instruments, as particles like electrons are so small and so numerous in a pumpkin (e.g., I estimate that there are at least $10^{30}$ particles in a pumpkin, which is a million times a trillion times a trillion).

If we add up a gazillion of these microscopic differences, all that will be left is ... nothing. The tree will still be there, and there will be grass around it, but there will be no pumpkin material whatsoever. So $S_{\text {gazillion }}$ isn't a situation with a pumpkin by the tree because there is absolutely nothing remotely pumpkinish in that scenario. The pumpkin claim evaluated with respect to that situation, $\mathrm{S}_{\text {gazillion, }}$ is false.

So at what point in the sequence of situations, the Ss, did there stop being a pumpkin by the tree? We started with a nice, healthy pumpkin and then considered a sequence of situations, each nearly identical to the one before in the sequence. Eventually, we had a situation with no pumpkin by the tree. So when did 'There is a pumpkin by the tree' go from just true to something else?

The sharpist says this: I don't know when the pumpkin claim goes from just true to something else, but I do hold that there's got to be two consecutive rows in which that's exactly what happens.

However, when faced with the question 'When did the pumpkin claim go from just true to something else?' nearly everyone will insist that there is something wrong with the question. The question is demanding a particular cutoff: for some number $n$, at situation $S_{n}$ there was a pumpkin by the tree (so the pumpkin claim was true when evaluated with respect to $S_{n}$ ), we then considered situation $S_{n+1}$, which differed from the previous section by one microscopic particle, and in that situation $S_{n+1}$ the pumpkin claim wasn't just true. But of course, we are inclined to say, there is no such sharp cutoff! Instead, we insist, the pumpkin goes away in a gradual manner, not all of a sudden. Imagine seeing a sequence of photographs of the pumpkinish thing, starting with $S_{1}$ and proceeding through $S_{2}$, $S_{3}$, and the rest in rapid succession. It would look like a pumpkin being very slowly destroyed from the top down, particle by particle. There would be no one point where you could say with any confidence 'Right 
there! That's the very point when the pumpkin no longer exists. An instant before it existed, but with that one particle gone it no longer exists'.

Yes, that is the entirely reasonable thing to say. No doubt about it. However, a compelling line of reasoning seems to prove that it's wrong. And no one knows what's wrong with the reasoning, if there's anything wrong with it at all.

We have a single claim, the pumpkin claim, evaluated at each of many very similar situations $S_{1}, S_{2}, S_{3}$, etc. The first situation involves a perfectly ordinary, full-grown, and healthy living pumpkin by the tree. Each subsequent situation differs from the previous situation by a miniscule difference. We end up with the following puzzling table:

\begin{tabular}{|c|c|}
\hline Situation & $\begin{array}{c}\text { Status of the } \\
\text { Pumpkin Claim }\end{array}$ \\
\hline $\mathrm{S}_{1}$ & True \\
\hline $\mathrm{S}_{2}$ & True \\
\hline $\mathrm{S}_{3}$ & True \\
\hline$\ldots$ & $\cdots$ \\
\hline $\mathrm{S}_{n}$ & $?$ \\
\hline$\ldots$ & $\cdots$ \\
\hline $\mathrm{S}_{\text {gazillion }-2}$ & False \\
\hline $\mathrm{S}_{\text {gazillion - 1 }}$ & False \\
\hline $\mathrm{S}_{\text {gazillion }}$ & False \\
\hline
\end{tabular}

What goes in the big open space in the second column-those zillions of rows? As soon as we have something other than 'true', proceeding from the top downwards, then we have our (first if not only) cutoff (cutoff $=$ change in status of the pumpkin claim). Even if some entries will be blanks, meaning that the pumpkin claim is neither true nor false, the first of those blanks (counting down from the top) will mark a cutoff. The pumpkin claim is true with respect to one situation and then has some other statusI don't care what-with respect to the very next situation. I'm not assuming that there is a cutoff, I'm arguing for it. The heart of the argument for a sharp cutoff is painfully simple: 
(a) In the first few trillion rows the correct entry is 'true'.

(b) The 'true' entries that start off from the top of the second column don't go in every row of that column (as it's clear that near the bottom the entries are 'false').

(c) So, there is some row when the top block of entries that read just 'true' stops in the sense that the entry in the next row isn't just 'true'. It could be something obvious like 'false'. Or, maybe it's 'neither true nor false'. Or, maybe it's 'meaningless'. Or, maybe it's something fancy like 'not true, not false, and not meaningless but $X^{\prime}$, for some exceedingly sophisticated and urbane $X$. In any case, that row marks a cutoff - that is, the pumpkin claim is just true with respect to the situation from one row, but the claim has some other status with respect to the situation in the next row-regardless of what appears in the next row (if anything at all).

Premise (a) certainly appears obviously and definitely true: surely $S_{1}, S_{2}, S_{3}, S_{4}$, and trillions more of the subsequent situations each contained perfectly good healthy pumpkins (again, the sum total of a few trillion of these changes wouldn't even be visible to the naked eye and wouldn't affect the functioning of the living pumpkin in any biological way), and 'There is a pumpkin by the tree', understood to have its perfectly ordinary meaning expressed in perfectly ordinary circumstances, was nothing other than just plain true with respect to those trillion or so situations. Thus, the first trillion or so rows in the second column of the table seem to get 'true' (or, if you like, 'true \& not false') put in them. That's premise (a).

There's simply no question that premise (b) is true, on literally any philosophical theory at all: no one is going to say that in situation $S_{\text {gaillion }}$ there is a pumpkin by the tree. Recall that at that point the pumpkin material has been completely removed.

In order to see why (c) seems to follow from (a) and (b), in the sense that if (a) and (b) are true then (c) has to be true as well, consider a different table in which the entries in the second column start out one way (so the analogy to premise (a) is true) but don't go on that way through the whole table (so the analogy to premise (b) is true too):

\begin{tabular}{|c|c|}
\hline Situation & $\begin{array}{c}\text { Numerical Status } \\
\text { of Pumpkin Claim }\end{array}$ \\
\hline $\mathrm{S}_{1}$ & 1 \\
\hline
\end{tabular}




\begin{tabular}{|c|c|}
\hline $\mathrm{S}_{2}$ & 1 \\
\hline $\mathrm{S}_{3}$ & 1 \\
\hline$\cdots$ & $\cdots$ \\
\hline $\mathrm{S}_{n}$ & $? ?$ \\
\hline$\cdots$ & $\cdots$ \\
\hline $\mathrm{S}_{\text {gaillion }-2}$ & 56 \\
\hline $\mathrm{S}_{\text {gaillion }-1}$ & 56 \\
\hline $\mathrm{S}_{\text {gaillion }}$ & 56 \\
\hline
\end{tabular}

You don't know what the numbers mean and you don't know what limitations there are on the numbers that can appear in the right column (e.g., whether there can be fractions like 7/8 in addition to whole numbers like 1 and 56). However, even when saddled with this ignorance a quick glance at the table is all you need to feel perfectly confident in concluding that the sequence of ' 1 's that starts the top of the right column has to end at some point, and thus there will be a pair of consecutive rows $X$ and $X+1$ such that row $X$ is the last one of the initial sequence of ' 1 's and in the next row $X+1$ there is something other than the simple ' 1 '. That's a sharp cutoff in numerical value. You have no idea whether at the first cutoff the numbers go from ' 1 ' to ' 56 ' or ' 1 ' to ' 2 ' or ' 1 ' to ' -34.45 ' or ' 1 ' to ' 1.0000000000001 ' or ' 1 ' to 'John Lee Hooker'. But you do know, immediately and with very little thought, that there simply must be a last row, counting down from the top, that has a simple ' 1 ' in it and the next row will have something else (or a blank).

Well, that's all premise (c) is saying: if the correct entries at the top of the column are 'true's, and the 'true's don't go throughout the whole column - that's premises (a) and (b) - then there simply must be a sharp cutoff: adjacent rows in which some row $S_{n}$ has just 'true' in it and the next row $S_{n+1}$ has something else in it. That's just logic.

It makes no difference for the existence of cutoffs (which is the thesis of sharpism) as to what goes in the row $S_{n+1}$ (if anything). Perhaps the entries in the second column of our table don't go from 'true' to 'false'. That is, maybe the claim made by your use of 'There is a pumpkin by the tree', when applied to situations $S_{n}$ and $S_{n+1}$ goes from true to indeterminate-or maybe to indeterminately indeterminate (or indeterminately indeterminately indeterminately ... indeterminate). Or maybe to just plain meaningless. Or maybe to both true and false (so it keeps being true but just adds falsity for some strange reason). Or 
maybe it goes from 'true $\&$ not $X$ ', for some status $X$ (let your creativity blossom here in wondering what $X$ might be), to 'true $\& X^{\prime}$ '. Or maybe its status with respect to $S_{n+1}$ changes with the wind, or my hair color, or some more likely factor. Or maybe it has no status whatsoever with respect to $S_{n+1}$ (not even meaningless). Or, what might not be any different, there might be no fact of the matter as to the status with respect to $S_{n+1}$ (whatever that idea comes to). Or perhaps it becomes incoherent to even apply the pumpkin claim to $S_{n+1}$. Finally, maybe the truth about the pumpkin claim with respect to $S_{n+1}$ is best captured by a Zen master's reaction to 'What is the sound of one hand clapping?'

One can think of some clever things to say regarding the status of the pumpkin claim when evaluated with respect to some rows; I've tried to give a hint of some of them immediately above. But one of the great strengths of the argument for sharpism is just this: it doesn't matter which of these many options one takes. Be as clever or as simple-minded as you like with your theory regarding the status of the pumpkin claim, it still seems inevitable that its status is ridiculously dependent on the minuscule difference of a single electron. The point is that 'There's a pumpkin by the tree', understood in the perfectly normal way, is true, meaningful, and not false when evaluated with respect to the first trillion or so situations, but at some point in the series of situations it stops having that status. That's all the sharpist is saying.

It's important to understand the thought experiment. For one thing, we are not changing the pumpkin over time: we are not imagining that the pumpkin in $\mathrm{S}_{1}$ loses a particle once a nanosecond, for instance, until it's entirely gone. $S_{2}$ doesn't come temporally after $S_{1}$; the pumpkin isn't rotting before our eyes. Instead, we are imagining a great many different situations each of which contains the whole episode of your visit to Farmer Fred's pumpkin patch. In each situation you pull up in your car with your niece, get out, and start walking on Farmer Fred's farm. And then your niece says 'Where are the pumpkins?' and you reply with the pumpkin sentence 'There is a pumpkin by the tree'. In $S_{1}$ she goes over to the tree and sees a nice full pumpkin; in $S_{\text {gazillion }}$ she goes over to the tree exactly as in $S_{1}$ and sees no pumpkin at all (and then complains to you that you were wrong). In certain intermediate situations she goes over to the tree and sees half a pumpkin (and then complains that the pumpkin isn't good enough for Halloween).

Thus, each situation is virtually identical in many relevant ways. For instance, in your mouth, 'There is a pumpkin by the tree' has the very same meaning in each possibility. The entire history of English is 
exactly the same, you are exactly the same, your linguistic, physical, and psychological history is exactly the same, your physical environment is almost exactly the same (just one particle difference each time!), and your interaction with your niece is exactly the same (until she goes around the shed to see the area by the tree).

I hear the following complaint: the table given earlier can't be completed! So, the thought goes, the problem never arises. Or maybe the complaint is this: it's indeterminate whether the table can be completed! Or maybe it's indeterminate whether it's indeterminate whether it can be completed, or.... But no: none of that matters. First, I (the sharpist) never said we could complete it. Second, I never even said that we either could or could not complete it. Third, I never said there was a unique correct answer for each row (more on that point below). Finally, on virtually anyone's view the first trillion or so slots in the second column can be completed: they all have nothing other than 'true' in them. Now you tell me: starting from the top, what is the last row we can correctly complete with just 'true'? The one trillionth trillionth row? Then that's our cutoff, and I couldn't care less what you want to say about the row after that one, no matter how philosophically sophisticated it is.

You might want to say, at some point in the table, 'We might as well stop at this point, although we could have stopped earlier'. But in the trillionth row for instance you could not have stopped putting in 'true'; that would have been just as much of a mistake as if you had stopped after the first row or the thousandth row. M aybe you think for some but not all rows we have a "genuine choice" as to putting in 'true'. Fine: when do we start to have such a "choice", since we obviously don't have such a "choice" in the first trillion rows? As soon as you say 'Okay, now we have a choice with the next row: we can put 'true' in it or we can put something else in it' we have our sharp cutoff.

When a very intelligent person is first exposed to this problem of vagueness-in the guise of the table above-usually they will have little hesitation in telling you about a million different ideas about various important philosophical notions such as reference, indeterminacy, the notion of definitiveness, context dependence, language use, the nature of truth, and meaning. This is the relatively easy part. M y advice: ask them (or yourself) how anything they just said applies to the individual entries in the right column of the pumpkin table given above. Is the top one true? Yes or no? What about the second one? Yes or no? The third? The last? Demand responses of them of these individual questions, even if the responses come as 'Well, that one is neither true nor false' or 'This one is true in some contexts of evaluation and 
false in others' or 'This one has no status at all' or 'This one neither has nor lacks a status' or even 'There is no answer to that one! You can't say anything about it!' The sharpist says that it just doesn't matter what fancy answers are offered: you can be as clever as you like with your high-minded ideas about what goes in the table and all she will do in response is point out that the column starts out with 'true' in it (the top rows), it doesn't have 'true' in all the rows, and, thus, there simply must be a first row, counting from the top, in which there is something other than just plain 'true' in the column. That's just inexorable logic; and that's a sharp cutoff because the pumpkin claim goes from true to something else with nothing more than the movement of a quark or electron. Until we get to the serious business of taking a stand on the status of the individual rows, and then seeing the consequences of those stands, discussion of vagueness is almost completely wasted. A person won't start productive work on the problem until she attempts to figure out what might show up in the second column of the table given above. Only then will she discover the true difficulty.

Suppose sharpism is true; would that be so bad?

The initial problem with sharpism is that we don't see anything in the sentence 'There is a pumpkin by the tree' that could make it just plain true when evaluated with respect to one situation $X$ and then false (or any status other than just plain true) when evaluated with respect to situation $Y$ even though the only difference between $X$ and $Y$ is the miniscule movement of an electron. For other sentences, the extreme sensitivity is understandable: 'There are 112 electrons in the vacuum chamber' could easily go from true to false with the removal of a single electron. But there is nothing in the pumpkin sentence to suggest it has such an exact meaning.

So, is there something in the context of utterance, say, that gives it such a discriminating meaning? We've never seen anything in linguistics or psychology or anywhere else that does the job as far as I know. Is there some exceedingly sharp line out there in nature dividing the pumpkins from the nonpumpkins that the pumpkin claim is attracted to, thereby giving it its sensitive cutoff? I know of no reason to think so; to think so is to believe in miracles; and keep in mind that we could replace the use of 'a pumpkin' in 'There is a pumpkin by tree' with 'something pumpkin-like' - and it's highly implausible that 'something pumpkin-like' has a perfectly exact meaning (even if you think that nature provides a perfectly sharp line for 'pumpkin' it's hard to see how it, with or without the help of facts about 
linguistic usage, could provide such a line for 'pumpkin-like', 'greenish', etc.). We are left with nothing to generate the sharp cutoff.

The vagueness paradox is this: although there is an extremely strong argument for sharpism, the truth of sharpism seems to require a linguistic miracle.

References

Horgan, Terrance. 1994. "Robust vagueness and the forced-M arch sorites paradox', Philosophical Perspectives, 8 (Logic and Language), 159-188.

Priest, Graham. 2003. "A Site for Sorites", in J. C. Beall (ed.), Liars and Heaps: New Essays on Paradox, Oxford: Oxford University Press, 24-38.

Williamson, Timothy. 1994. Vagueness, London: Routledge. 